NASA/TM- $-\varepsilon^{\prime} /-207541$
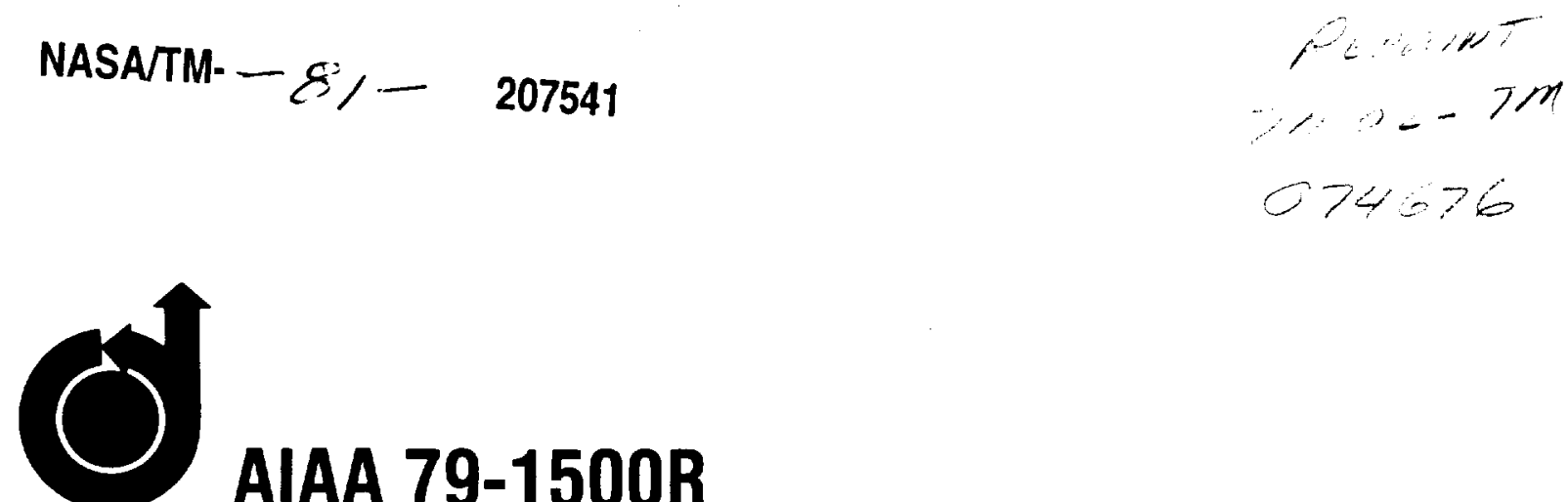

AIAA 79-1500R

Transonic Flow Past a Symmetrical Airfoil at High Angle of Attack

D. A. Johnson, W. D. Bachalo, F. K. Owen 


\title{
Transonic Flow Past a Symmetrical Airfoil at High Angle of Attack
}

\author{
D. A. Johnson," W. D. Bachalo, $†$ and F. K. Owen \\ NASA Ames Research Center, Moffett Field, Calif.
}

\begin{abstract}
The results of an experimental investigation of shock-induced stall and leadingedge stall on a 64A010 airfoil section are presented. Advanced nonintrusive techniques-laser velocimetry and holographic inteferometry - were used in characterizing the inviscid and viscous flow regions. The measurements include Mach contours of the inviscid flow regions, and mean velocity, flow direction, and Reynolds shear stress profiles in the separated regions. The experimental observations of this study are relevant to efforts to improve surfacepressure prediction methods for airfoils at or near stall.
\end{abstract}

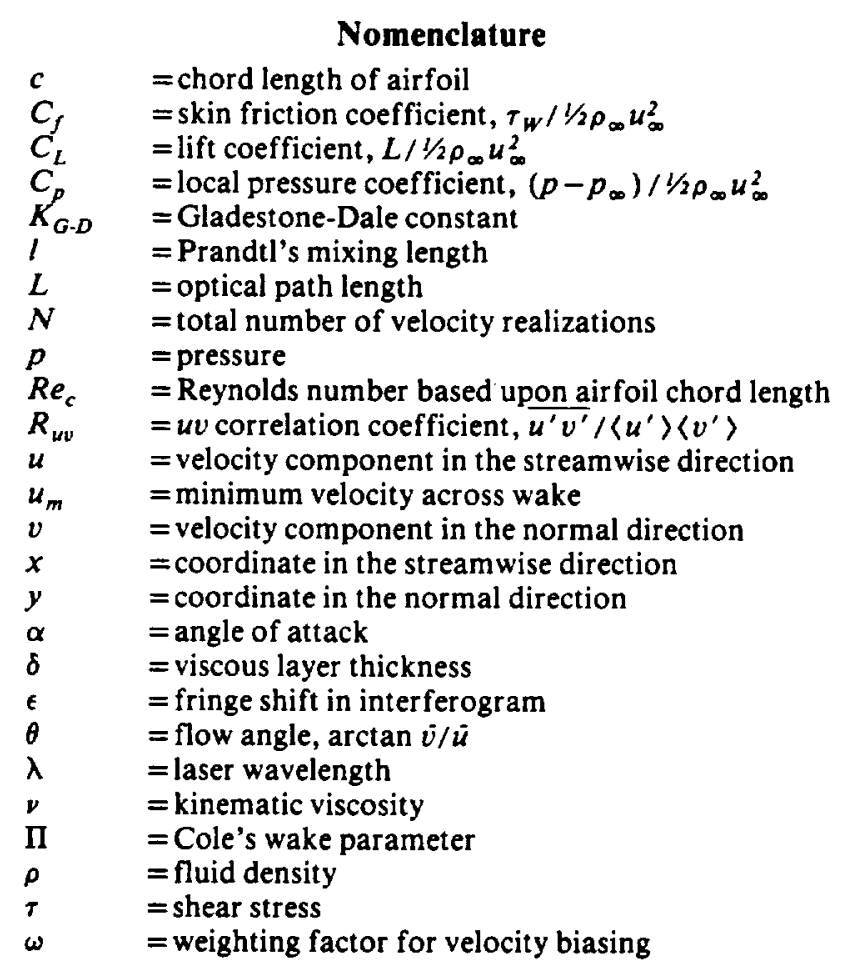

Subscripts

e $\quad=$ conditions at edge of viscous layer

$i \quad=i$ th velocity realization

$s \quad=$ surface

$w \quad=$ conditions at the surface

$\infty \quad=$ freestream conditions

\section{Superscripts}

$$
\begin{aligned}
& \text { (') = fluctuating quantity } \\
& \text { (-) = time-averaged quantity } \\
& \text { (') = rms value of quantity }
\end{aligned}
$$

Presented as Paper 79-1500 at the AIAA 12th Fluid and Plasma Dynamics Conference, Williamsburg, Va., July 23-24, 1979; submitted Sept. 5, 1979; revision received April 11, 1980. This paper is declared a work of the U.S. Government and therefore is in the public domain.

- Research Scientist. Member AIAA.

$\dagger$ Staff Scientist, Spectron Development Laboratory, Costa Mesa, Calif. Member AIAA.

‡Consultant, Complere, Inc., Palo Alto, Calif. Member AIAA.

\section{Introduction}

$\mathbf{V}$ IRTUALLY every conceivable lifting situation occurs on the blades of a helicopter rotor. Conditions of attached flow and high-lift efficiency, shock-induced separation (advancing blade tip), and leading-edge separation (retreating blade) are encountered during each revolution. The situation is further complicated by the time dependency and threedimensionality of the flow. To address these complicated flows, a better understanding is needed of two-dimensional, nontime-dependent flow cases. Presently, prediction methods are unable to predict the flow accurately for steady, twodimensional stall due to shock-induced or leading-edge separation. This is due largely to inaccurate turbulence models for the turbulent separated viscous layer and the near wake, a consequence of the paucity of experimental information. Measurements of the transport properties of these highly turbulent separated flows is needed to develop improved turbulence models and hence better prediction methods. In the past, these measurements were difficult to make, but recent development of the laser velocimeter has made the problem more tractable.

In the present paper, experimental data are examined for the cases of shock-induced and leading-edge stall on an NACA $64 A 010$ airfoil section providing new insights into these two flow phenomena. Several diagnostic techniques were used to quantify the flow behavior: 1) laser velocimetry to provide measurements of the mean flow velocities (streamwise and normal) and the turbulent transport properties; and 2) holographic interferometry to provide a complete description of the density field from which the Mach number and static pressure fields in the inviscid flow were deduced.

The present paper is an extension of the work presented in Ref. 1. Flowfield results are presented for $\alpha=8$ deg at $M_{\infty}=0.8$, a more severe shock-induced separation than studied in Ref. 1 , and for $\alpha=12 \mathrm{deg}$ at $M_{\infty}=0.7$, a condition with leading-edge separation. Also, flow directions in the near wake, which were not addressed in Ref. 1, are presented. These data reveal a strong entrainment process at the trailing edge that results in rapid closure of the wake.

Very few flowfield measurements have been made for airfoils under condition of separated flow, primarily because of the experimental difficulties associated with separated flows. At low speeds Seetharam and Went ${ }^{2}$ explored the case of trailing-edge stall on a $17 \%$ thick $\mathrm{GA}(\mathrm{W})-1$ airfoil section using a variety of pressure probes to measure local total and static pressure and flow direction. Measurements were not made of the turbulent transport properties. Recent results obtained using a "flying" $x$-array hot-wire probe have been reported by Coles and Wadcock ${ }^{3}$ for a NACA 4412 airfoil 
section at maximum lift. In this study, hot-wire probes were swung in a circular arc to prevent reversed velocities relative to the probe. These results include mean flow velocities, flow direction, and the turbulent transport properties, including the Reynolds shear stresses. This experimental method would not be practical for high-speed applications due to the prohibitive speed at which the hot-wire probe would have to be swung. There is also concern regarding probe interference effects. No analysis has yet been reported on these data with regard to turbulence modeling.

Measurements at higher Mach numbers (0.15 and 0.5) using a laser velocimeter system have been made for an NACA 0012 airfoil section at stall conditions by Young and Hood. ${ }^{4}$ The measurements were confined to regions well removed from the airfoil surface (especially at the higher Mach number). Reported turbulent flow properties consisted of only the streamwise turbulence intensity at the low Mach number condition. At transonic conditions, two airfoil studies ${ }^{1,5}$ have been performed wherein separated flow measurements were obtained. In the study of Seegmiller, Marvin, and Levy, 5 laser velocimeter measurements were obtained on an $18 \%$ thick circular arc airfoil at zero angle of attack for Mach numbers of 0.76 and 0.79 . At the higher Mach number, shock-induced separation occurs at about $60 \%$ chord. At the lower Mach number, an unstable flow condition is established as separation alternates between the upper and lower surface; the flow is accompanied by extensive shock wave motion. Mean velocities and turbulent transport properties, which include the Reynolds shear stress, were measured in the near wake and along the aft $20 \%$ of the section. The second study was of an airfoil under lifting conditions at transonic speeds. That investigation was conducted by two of the present authors (Johnson and Bachalo ${ }^{1}$ ). In that study, mean velocity and turbulent flow measurements were obtained on a NACA 64A010 airfoil section (the same model as used in the present study) for $M_{\infty}=0.8$ and for three angles of attack: $0,3.5$, and 6 deg. The flow conditions ranged from an attached flow to a severely separated (shock-induced) flow. As in the present study, laser velocimetry and holographic interferometry were employed in that investigation. Viscous flow measurements included mean velocities and turbulent transport properties including turbulent shear stresses for the near wake and along the aft one-third of the airfoil's upper surface.

A number of basic investigations have been performed on transonic shock-wave/turbulent boundary-layer interactions (e.g., Refs. 6-10). However, with the flow models used in those studies (of which all used contoured-wall models, with the exception of the model in Refs. 9 and 10), there was no wake influence on the flow as there would be with an airfoil.

\section{Experimental Technique}

Experimental procedures were essentially the same as those used in Ref. 1. Because they are covered in detail in Ref. 1, they are only briefly discussed here. The test facility was the Ames 2 by $2 \mathrm{ft}$ Transonic Wind Tunnel, which is a closedreturn, variable-density tunnel with $21 \%$ open porous-slotted upper and lower walls for transonic testing. A 6 in. chord section, which spanned the entire test section, was used. It was attached at its end points to the tunnel Schlieren windows by pin mounts. Angle-of-attack changes were accomplished by rotation of those windows. The chord Reynolds number for all conditions was $2 \times 10^{6}$. Transition strips were affixed to the airfoil section at the $17 \%$ chord station on the upper and lower surfaces to insure that the boundary layer was turbulent at the foot of the shock wave for all $M_{\infty}=0.8$ conditions. The effectiveness of the strips was verified in the study of Ref. 1. For the $M_{\infty}=0.7, \alpha=12$ deg case, in which separation occurred upstream of the upper surface strip, the transition strips undoubtedly had little effect on the overall flow behavior.

\section{Laser Velocimeter}

The laser velocimeter system discussed in Ref. 1 was used in the present study. This system is capable of simultaneous twovelocity component measurements. Bragg cell frequency shifting, as needed to explore separated and highly turbulent flows, was incorporated in both the streamwise and vertical velocity set of optics. The effective sensing volume of the system was approximately that of a cylinder, $200 \mu \mathrm{m}$ in diameter and $3 \mathrm{~mm}$ long, whose axis was aligned with the cross-stream direction.

Signal processing was accomplished with single-particle burst counters, and the individual realizations from the two channels were simultaneously recorded with a digital computer. This allowed the velocity correlation, $\bar{u}^{\prime} v$, to be obtained in a straightforward manner by multiplying and averaging. The mean velocities, turbulence intensities, and velocity correlations, $\overline{u^{\prime} v^{\prime}}$, were calculated as follows:

$$
\begin{aligned}
\bar{u} & =\frac{\sum_{i=1}^{N} \omega_{i} u_{i}}{\sum_{i=1}^{N} \omega_{i}} \\
\left\langle u^{\prime}\right\rangle & =\left(\frac{\sum_{i=1}^{N} \omega_{i} u_{i}^{2}}{\sum_{i=1}^{N} \omega_{i}}-\bar{u}^{2}\right)^{k} \\
\overline{u^{\prime} v^{\prime}}= & \frac{\sum_{i=1}^{N} \omega_{i} u_{i} v_{i}}{\sum_{i=1}^{N} \omega_{i}}-\overline{u v}
\end{aligned}
$$

In Eqs. (1-3), $\omega_{i}$ is the weighting factor used to account for particle arrival rate dependency on instantaneous velocity. The two-dimensional weighting factor

$$
\omega_{i}=\frac{I}{\sqrt{u_{i}^{2}+v_{i}^{2}}}
$$

as suggested in Ref. 11 was adopted. The equations for $\bar{v}$ and $\left\langle v^{\prime}\right\rangle$ are identical to Eqs. (1) and (2), except that $v_{i}$ is used instead of $u_{i}$.

At each point in the flow, at least several thousand velocity realizations were used to calculate the flow properties given in Eqs. (1-3). Naturally occurring particles in the tunnel were used as light scatterers for the velocimeter. In this facility, lubrication oil within the drive system vaporizes and later condenses in the tunnel circuit to provide a generous supply of scattering centers. Previous measurements ${ }^{9}$ across a normal shock have shown that these particles are small enough (estimated to be $1 \mu \mathrm{m}$ ) to give very good response to a step change in velocity at sonic speeds.

\section{Holographic Interferometer}

The holographic techniques described in Ref. 1 were also used in the present study. The double-plate method ${ }^{12}$ was used with the existing tunnel Schlieren mirrors, as part of the optical train. Infinite-fringe interferograms were reconstructed from the no-flow plate and the plates taken at the desired test conditions. In the interpretation of these interferograms, the flow was assumed to be two-dimensional. The validity of this assumption was enhanced by the model aspect ratio of 4 . Under the assumption of two-dimensional flow each fringe of the interferogram corresponds to a line of constant density and the change in density between adjacent 
fringes is given by

$$
\Delta \rho=\frac{\lambda}{L} \frac{I}{K_{G \cdot D}}
$$

Once the density at one point in the flow is established, the remaining contours can be determined from Eq. (5). As seen from Eq. (5), the larger the span of the tunnel, which corresponds to $L$ in Eq. (5), the better the resolution of the interferogram. In this facility, one fringe shift at $M_{\infty}=0.8$ corresponds to a change of only about $0.5 \%$ in density and a corresponding change in Mach number of $1 \%$.

\section{Results and Discussion}

\section{Inviscid Flow and Surface Pressure}

Infinite-fringe interferograms obtained by holographic interferometry for the two different types of stall are presented in Fig. 1. The reader is reminded that each fringe represents a line of constant density. Under the assumption of isentropic flow, these fringes also represent lines of constant Mach number. Readily apparent from these interferograms is the very thick viscous layers that have developed as a result of massive separation. In the shock-induced stall condition, the viscous layer is about $20 \%$ of the chord at the trailing edge; in the leading-edge stall condition it is twice as thick. For the leading-edge stall case, there is no apparent vortical pattern as would be expected if discrete shedding were occurring from the leading edge. In the shock-induced stall interferogram, the shock wave appears relatively thick (about $6 \%$ chord). This is believed to be a result of three-dimensional effects. Near the side walls of the tunnel the shock wave is likely further forward as a consequence of the sidewall shock-wave/boundarylayer interactions. In the interferograms presented in Ref. 1, which were at lower angles of attack, this apparent broadening of the shock wave was significantly less. Obviously, greater three-dimensionality in the flow should be expected at this higher angle of attack. Notice that there is not a lambda shock pattern.

In Fig. 2, the lifting characteristics of this airfoil $\left(C_{L}\right.$ vs $\left.\alpha\right)$ at $M_{\infty}=0.7$ and 0.8 are shown for the present test configuration. For both of the present experimental cases, the angle of attack is well beyond the region where the lift curve is linear. In the shock-induced case the lift is still increasing with angle of attack, although at a low rate. Angles of attack greater than 8 deg were not studied due to the concern that a transitional boundary layer at the foot of the shock wave could result if the shock position was too close to the transition strip. The shock-induced case could probably be more accurately classified as an incipient stall case, in contrast to the leading-edge separation test condition, in which the airfoil is definitely stalled.

Quantitative data other than density can be obtained from the interferograms if certain assumptions are made about the flow. For instance, if it is assumed that the flow is isentropic in the inviscid regions, local Mach number and static pressure can be obtained. In the region downstream of the shock wave, where the flow is rotational, this assumption can still be used if the total pressure losses across the shock wave are sufficiently small. In Fig. 3, measured surface pressures, and pressures obtained at the edge of the viscous layers from the interferograms of Fig. 1, are presented. For the leading-edge separation case, the edge of the shear layer near the leading edge is not well defined in the interferogram due to the steep gradients in the inviscid flow; because of the lack of definition, upper surface shear layer edge pressures are not presented upstream of $40 \%$ chord for this case. For the shockinduced stall case, account was taken of the total pressure loss across the shock wave. This was done by calculating the total pressure loss at the foot of the shock wave from the upstream Mach number and shock inclination, and assuming that the total pressure was constant along the edge of the viscous
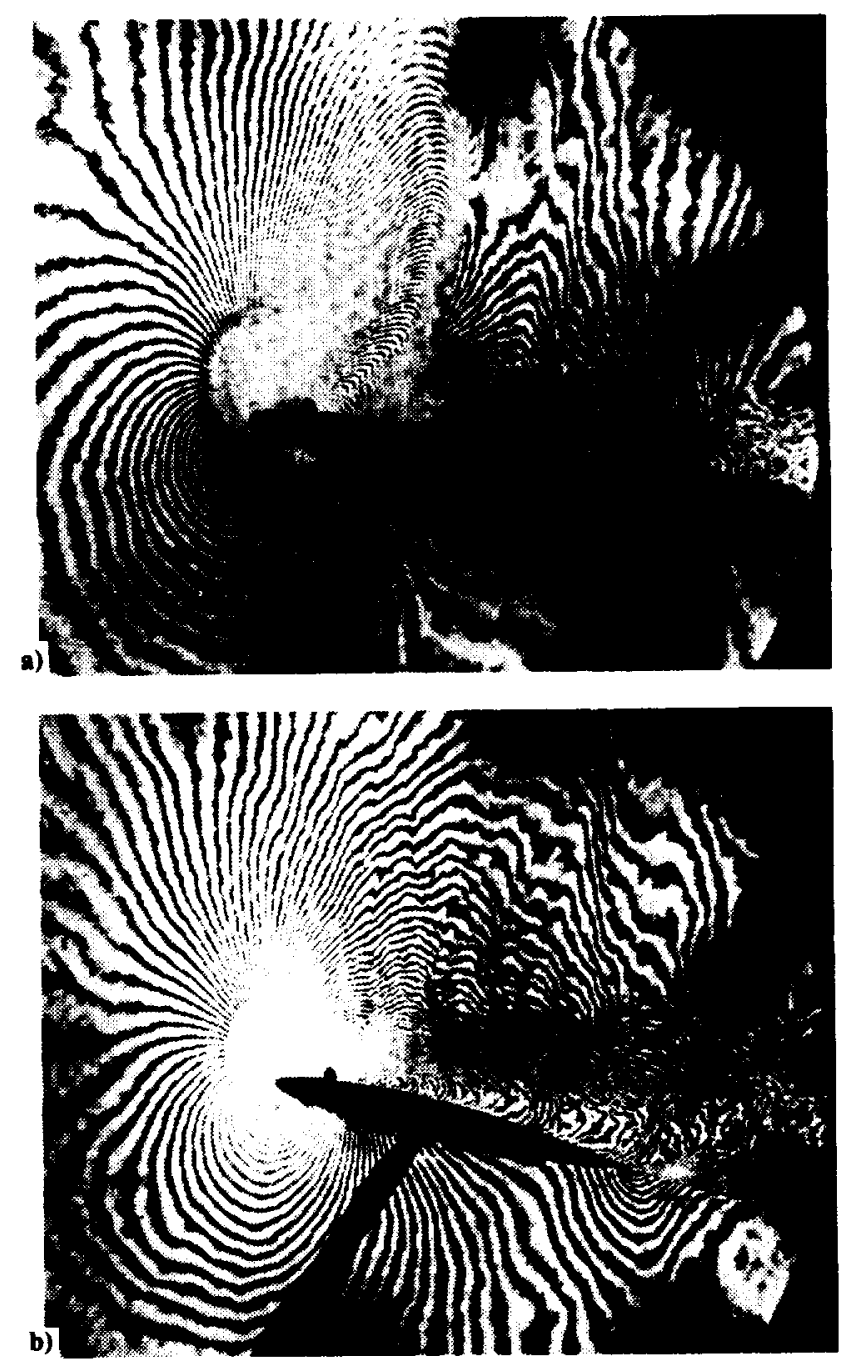

Fig. 1 Infinite-fringe interferograms: a) $M_{\infty}=0.8, \alpha=8$ deg; b) $M_{\infty}=0.7, \alpha=12 \mathrm{deg}$.

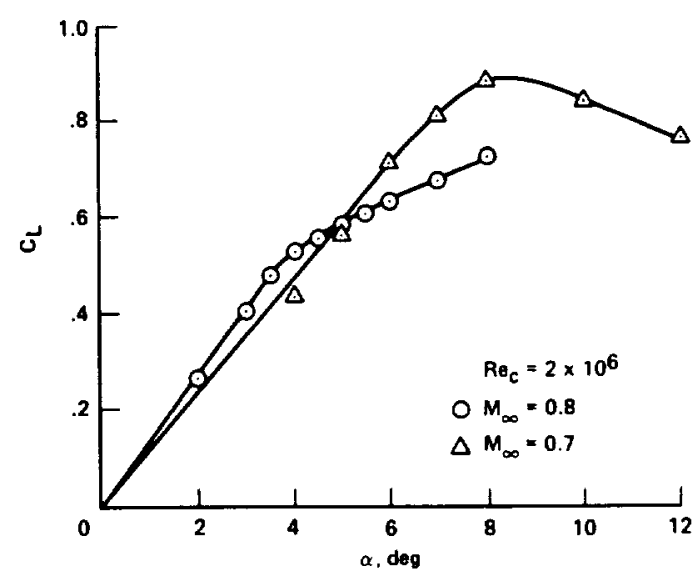

Fig. 2 Lifting characteristics of NACA $64 A 010$ for lest configuration of present study.

layer. The pressure comparisons are made in Fig. 3 to demonstrate that the static pressure differences across the viscous layers and the near wake are small. Edge velocity measurements obtained with the laser velocimeter at the trailing edge also showed the edge static pressure within experimental accuracy to equal the surface static pressure. The equal trailing-edge pressures demonstrate that the Kutta condition is still valid even for these severely separated cases. A closer examination of the interferograms in Fig. I shows 

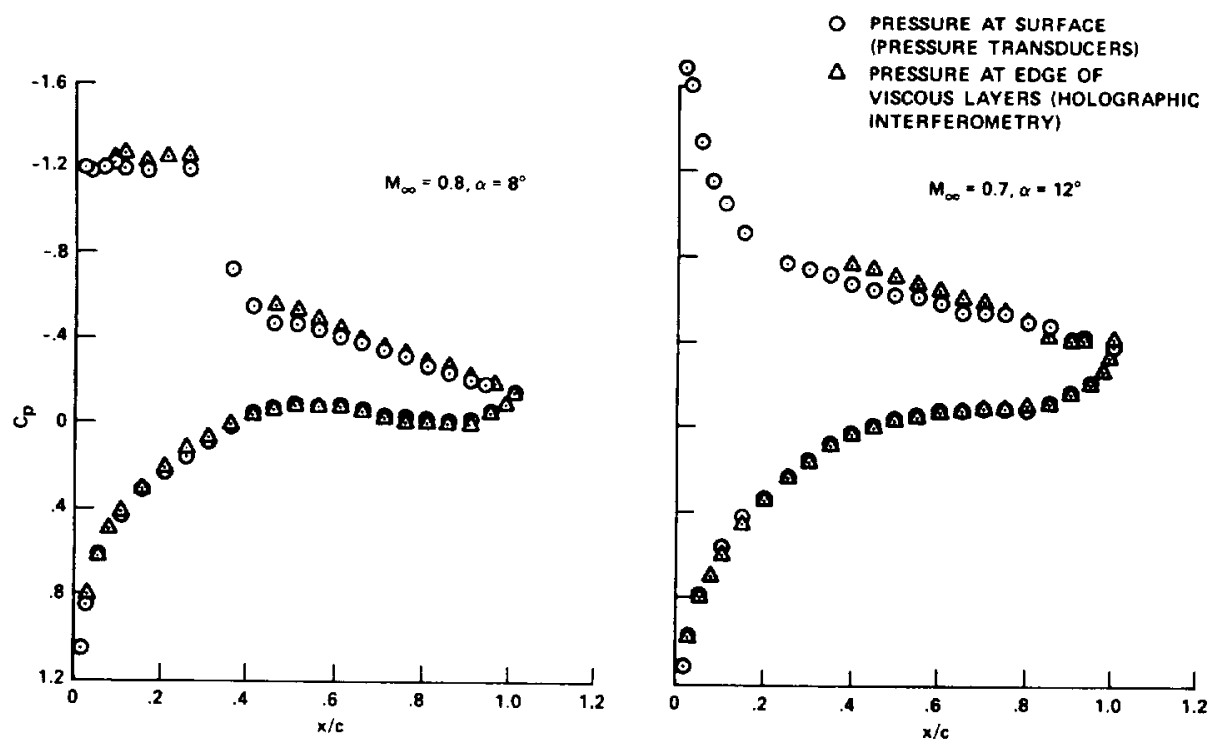

Fig. 3 Comparison of boundary-layer edge pressures with surface pressures.
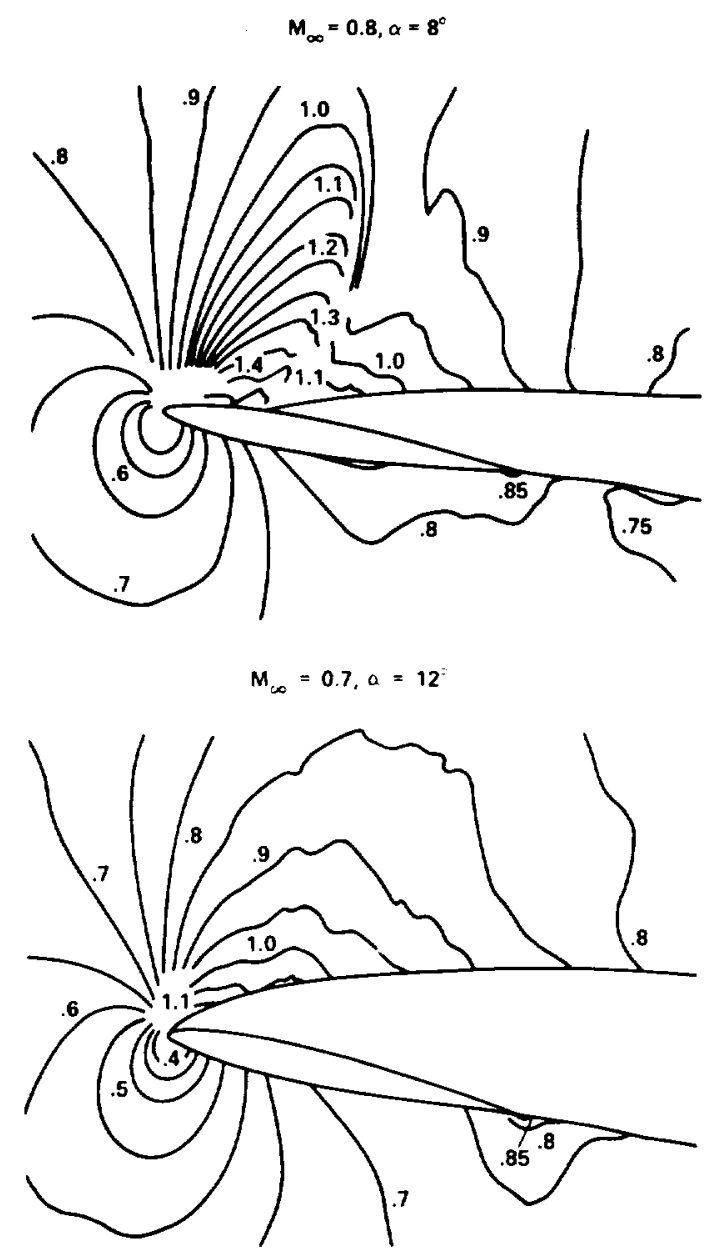

Fig. 4 Mach number contours determined from infinite-fringe interferograms.

that a rapid increase in speed does occur along the lower surface at the trailing edge as demanded by the Kutta condition.

Mach contours obtained from the interferograms are shown in Fig. 4. No account was taken of shock losses in obtaining these contour plots. Lines of constant density were interpreted as lines of constant Mach number. The maximum error in Mach number caused by ignoring these losses is 0.025 .

\section{Mean Viscous Flow Properties}

Laser velocimeter results for the mean streamwise velocity and the flow angle near the trailing edge of the airfoil $(x / c=1.03)$ are presented in Fig. 5 for both test cases. The vertical distance is referenced from the trailing edge. Included in Fig. 5 are results obtained for $M_{\infty}=0.8$ and $\alpha=5 \mathrm{deg}$. For the leading-edge separation case, as seen from Fig. 5 , the minimum streamwise velocity is only slightly negative $\left(u / u_{e} \approx-0.01\right)$; and for the strong shock-induced separation, it is just slightly positive $\left(\dot{u} / u_{e} \approx+0.02\right)$ at this streamwise station which is about $0.5 \mathrm{~cm}$ downstream of the trailing edge. For the milder separation $(\alpha=5 \mathrm{deg})$, an increase in the minimum velocity has already occurred. At $\alpha=5$ deg, data were taken sufficiently low in $y$ that the large strain rate of the lower surface boundary layer is evident. In all three cases, the minimum velocity occurred above the trailing edge of the airfoil.

The most striking feature in the local flow angle behavior is the near vertical downward flow direction about the point of minimum streamwise velocity for the massive separation cases. The large negative flow angles at the trailing edge indicate that very rapid entrainment of the slow-moving fluid above the airfoil's upper surface into the high-energy lower surface boundary layer is taking place. This flow character will be treated in more detail by observing the development of $\theta$ with streamwise distance from the trailing edge, but first the nature of the mean streamwise velocity profiles will be examined.

As seen from Fig. 5, the mean velocity profiles for both $M_{\infty}=0.8, \alpha=8 \mathrm{deg}$ and $M_{\infty}=0.7, \alpha=12 \mathrm{deg}$ have the shape of a free shear layer. To assess the similarity in the mean velocity profiles, the data in Fig. 5 were replotted with $\bar{u} / u$. and $y / \delta$ as the parameters in Fig. 6 . Included in Fig. 6 is Cole's ${ }^{13}$ theoretical profile for $u_{r}=0\left(\bar{u} / u_{e}=\sin ^{2} \pi / 2 y / \delta\right)$. Van-Driest transformed velocities have not been used because the edge Mach numbers are sufficiently low that the effect of this scaling is negligible. Plotting the mean velocities in this manner collapses the data, and Cole's wake function seems to fit both sets of data reasonably well (Fig. 6). In the study of Ref. 1 , it was found that for $M_{\infty}=0.8, \alpha=6 \mathrm{deg}$ the mean velocity profiles obtained on the airfoil's surface were similar to those obtained just downstream of the trailing edge with the reversed flow velocities being very low. Similar to the $\alpha=8$ deg case the flow in the $\alpha=6$ deg case remains separated from the foot of the shock wave to the trailing edge. These data of Ref, 1 are shown in Fig. 7. The profiles obtained at $x / c=1.02$ and 0.83 also fit Cole's theoretical profile relatively well for $u_{r}=0$. This is not true for the profile at $x / c=0.67(0.30$ chord downstream of the separation point). At this station, a better 


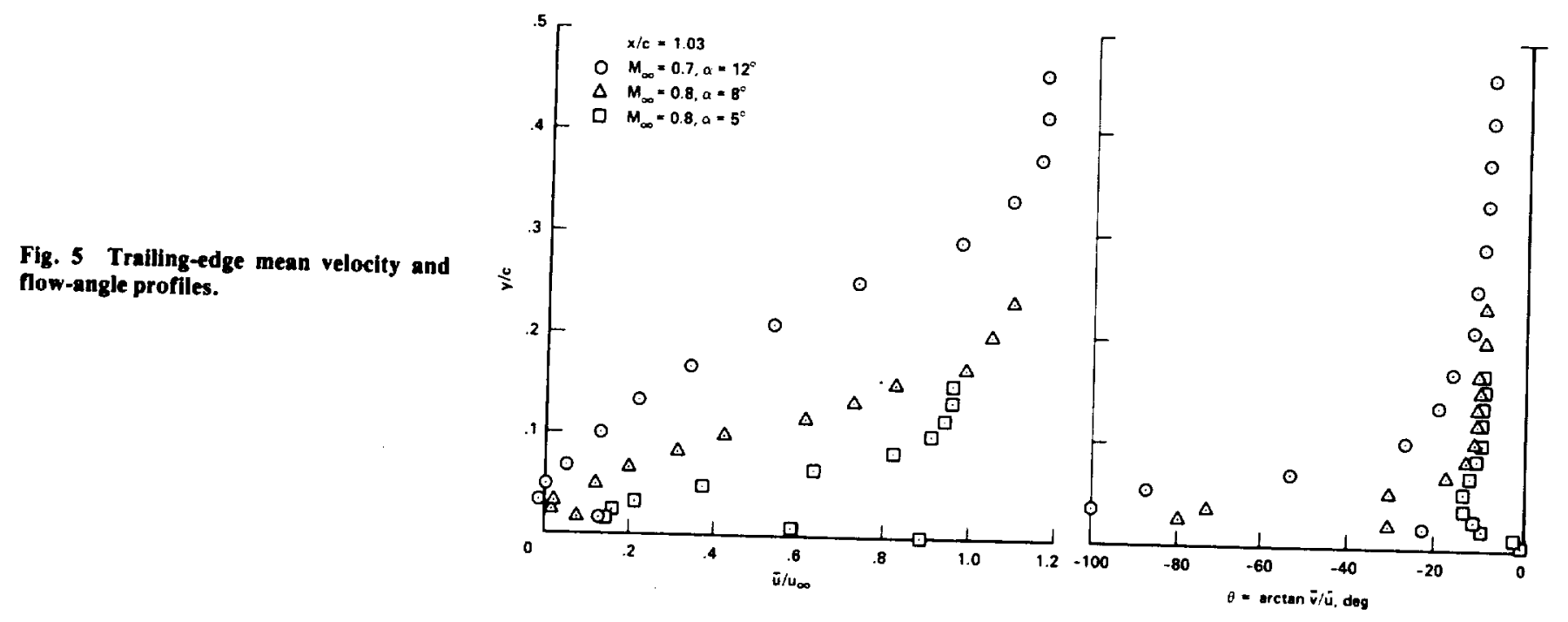

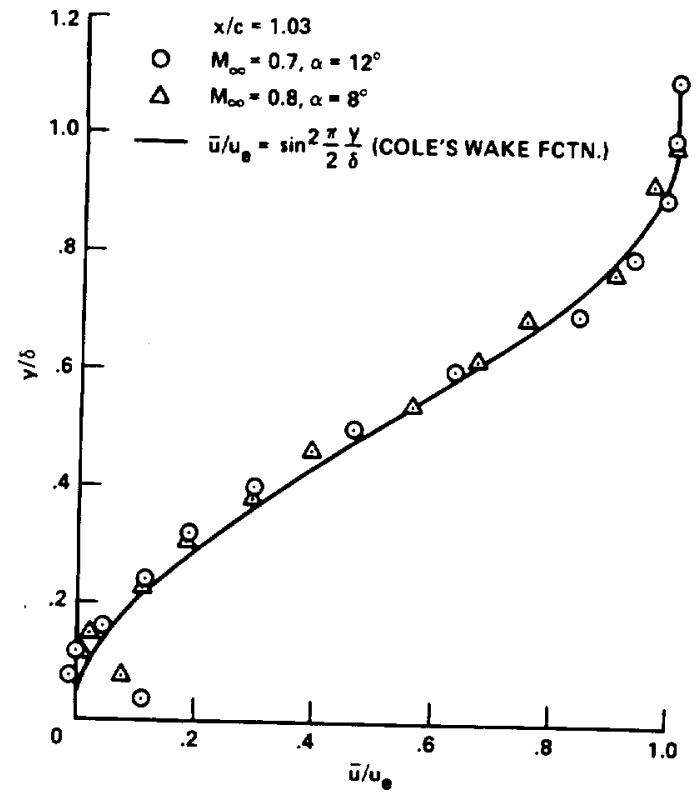

Fig. 6 Trailing-edge mean velocity profiles scaled to boundary-layer thickness and edge velocity.

fit was obtained with Cole's theoretical profile for a nonzero value of $u_{\text {r }}$

$$
\frac{\bar{u}}{u_{e}}=\frac{u_{\tau}}{u_{e}}\left\{\frac{1}{0.41} \ln \left[\frac{y}{\delta}\left(\frac{u_{\tau} \delta}{\nu}\right)\right]+s\right\}+\frac{u_{\tau}}{u_{e}} \frac{\Pi}{0.41} \sin ^{2} \frac{\pi}{2} \frac{y}{\delta}
$$

which can be shown ${ }^{13}$ to reduce to $\bar{u} / u_{e}=\sin ^{2}(\pi / 2)(y / \delta)$ when $u_{r}=0$. However, only a very small value for $u_{r}$, had to be assumed (i.e., $u_{T}=-0.005 u_{e}$ which corresponds to a skin friction coefficient $C_{f}$ of $\left.-0.5 \times 10^{-4}\right)$. The agreement between the $\alpha=6$ deg data and Eq. (6) with an assumed slightly negative or zero value for $u$, suggests that the wall shear is very low in the separation zone. It is believed that similar wall conditions are present for the $\alpha=8 \mathrm{deg}, M_{\infty}=0.8$ amd $\alpha=12 \mathrm{deg}, M_{\infty}=0.7$ cases

Near-wake velocity recovery and flow-angle development with streamwise distance for $M_{\infty}=0.8, \alpha=6 \mathrm{deg}$ are shown in Fig. 8. Overall, the flow-angle measurements indicate a significantly different flow pattern from that suggested for the $\mathrm{GA}(\mathrm{W})-1$ cusped, trailing-edge airfoil. ${ }^{14}$ In that model (Fig. 9a), the flow at the trailing edge is postulated to have an S-shaped pattern, with fluid from the lower surface boundary layer flowing into the upper surface separated zone, being transported upstream in the separated zone, and then being convected downstream along a path close to the dividing streamline. The present results indicate no net flow of fluid from the lower surface boundary layer into the separation bubble. Instead, there is a net downward flow of the slowmoving fluid above the airfoil's upper surface into the lower surface viscous layer as a result of entrainment. The differences observed in the near-wake flow character may be a result of the radical difference in airfoil geometry. The $64 \mathrm{A010}$ section is a $10 \%$ thick, symmetrical airfoil with a sharp trailing edge (the aft $30 \%$ of the airfoil is essentially a wedge with a 6 deg half-angle), whereas the $G A(W)-1$ airfoil section is a $17 \%$ thick cambered airfoil with a blunt trailing edge. The present results indicate a flow situation as depicted in Fig. 9b. At the trailing edge, the fluid in the "dead zone" just above the trailing edge is entrained into the high-velocity lower stream (the lower surface boundary layer is extremely thin, as it is in a simple single-stream mixing experiment) resulting in flow angles near $90 \mathrm{deg}$. Further downstream, due to mixing with the lower surface high-energy layer, the flow takes on a wake character with the minimum mean streamwise velocity occurring above the trailing edge of the airfoil. In this region, the displacement thickness of the wake is rapidly decreasing, causing inward flow at the upper and lower wake interfaces relative to the outer inviscid flow. To obtain reasonable predictions of the near-wake development, it appears that this entrainment process will have to be taken into account.

\section{Turbulent Flow Properties}

The turbulence intensities and Reynolds shear stresses (i.e.

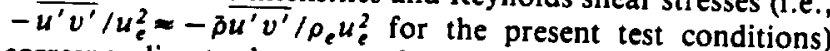
corresponding to the mean velocity profiles of Fig. 6 are given in Figs. 10 and 11 , respectively. As seen in these figures, the turbulence properties at the trailing edge of the airfoil differ very little for these two types of stall. Notice that the shear stress changes sign when the strain rate $\partial \dot{u} / \partial y$ changes sign at the lower edge of the layer, as the eddy viscosity formulaticn predicts. The similarities in turbulence distributions are not that surprising if one considers both viscous layers to be fully developed turbulent shear layers. The levels of turbulence and shear, however, are different from those observed in lowspeed, free-shear-layer experiments, such as those of Liepmann and Laufer ${ }^{15}$ and Spencer and Jones. ${ }^{16}$ The turbulence levels in both the streamwise and vertical directions are about $50 \%$ higher than those of Spencer and Jones. As a result, the maximum shear stress is approximately twice as high. The maximum correlation coefficient, $R_{u v}=u^{\prime} v^{\prime} /\left\langle u^{\prime}\right\rangle\left\langle v^{\prime}\right\rangle$. 


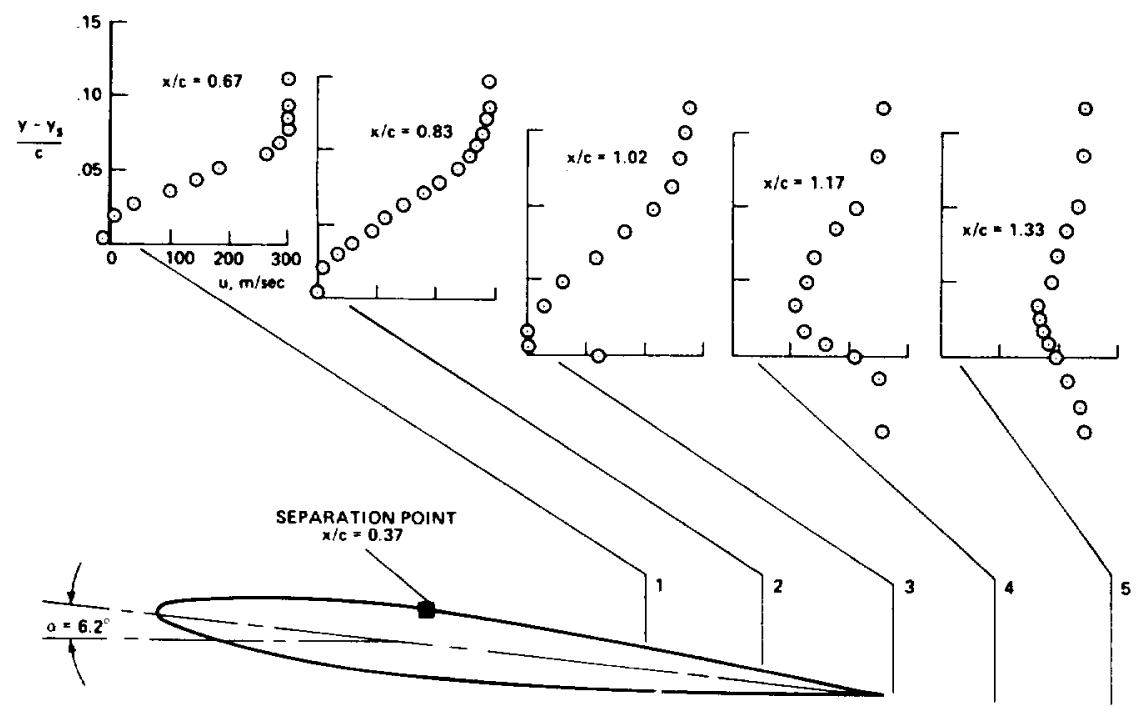

Fig. 7 Mean velocity profiles for $M_{\infty}=0.8$, $\alpha=6$ deg (Fig. 11 of Ref. 1).

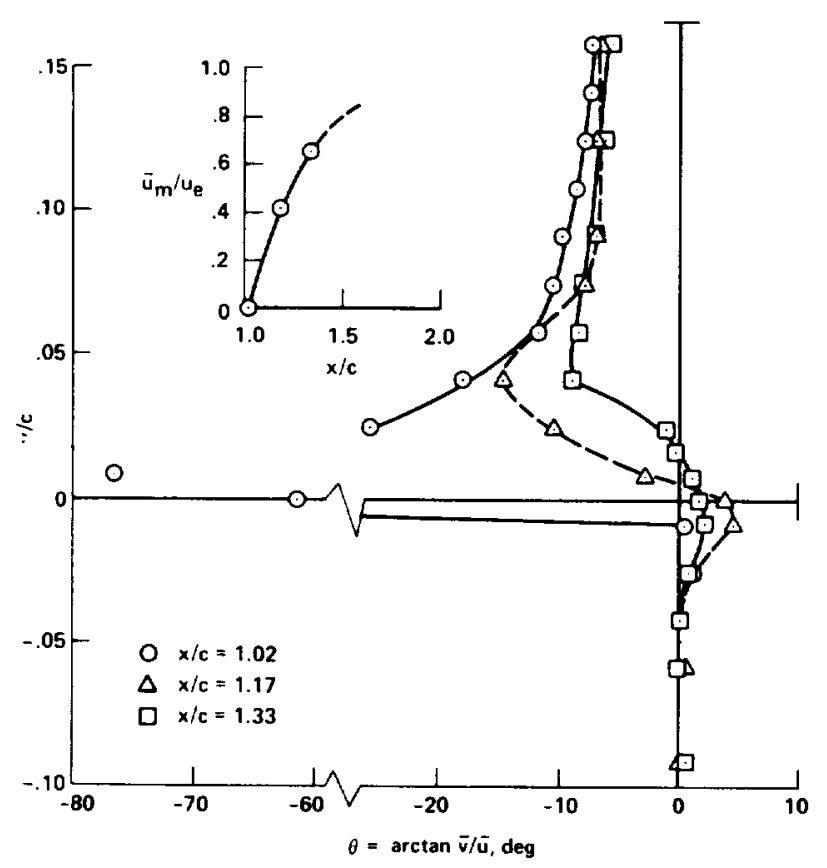

Fig. 8 Flow-angle development in near wake, $M_{\infty}=0.8, \alpha=6$ deg.

ranged between -0.5 and $-0.62 ;$ Spencer and Jones gave a value of -0.54 .

Unexpectedly, the maximum value attained in the nondimensional shear stress, $\left(-\overline{u^{\prime} v^{\prime}} / u_{e}^{2}\right)_{\max }$, for $\alpha=5$ deg (the mean velocity profile for this case was presented in Fig. 5) was nearly the same as that observed for the two stall cases. A plot of this quantity for $x / c \approx 1.03$ vs angle of attack is presented in Fig. 12 (data from Ref. 1 have been included in this figure). As seen from Fig. 12, $\left(-\overline{u^{\prime} v^{\prime}} / u_{e}^{2}\right)_{\max }$ at the trailing edge remains nearly constant for angles of attack of 5 deg and greater. Moreover, it is nominally constant upstream of the trailing edge for $\alpha=6 \mathrm{deg}$ (the only case for which data have been obtained for $x / c<1)$.

The behavior of $\left(-\overline{u^{\prime} v^{\prime}} / u_{e}^{2}\right)_{\max }$ with change in angle of attack provides some insight into the progression of separation on this airfoil. Oil flow observations indicate the flow to be attached at $\alpha=0$ and 2 deg but separated at the foot of the shock wave at $\alpha=3.5 \mathrm{deg}$. At this angle of attack, the shock wave reaches its furthest aft position ( $50 \%$ chord). As the angle of attack is increased further, the shock wave starts to move forward, indicating the presence of separation. It is

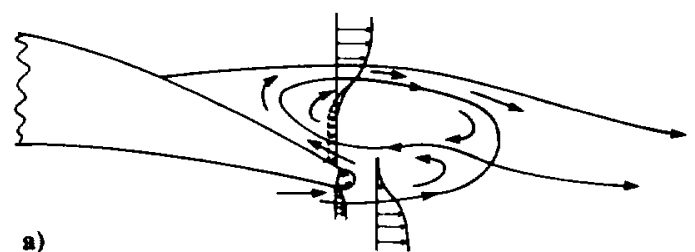

STREAMWISE VELOCITY

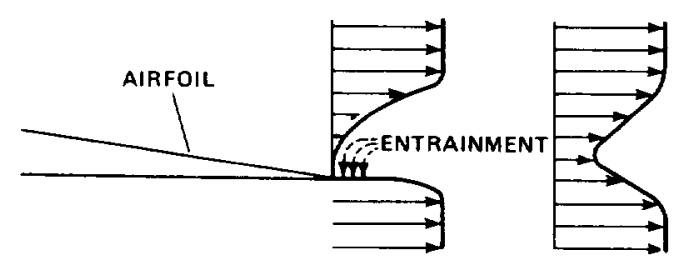

FLOW ANGLE, $\theta=\arctan \bar{v} / \bar{u}$

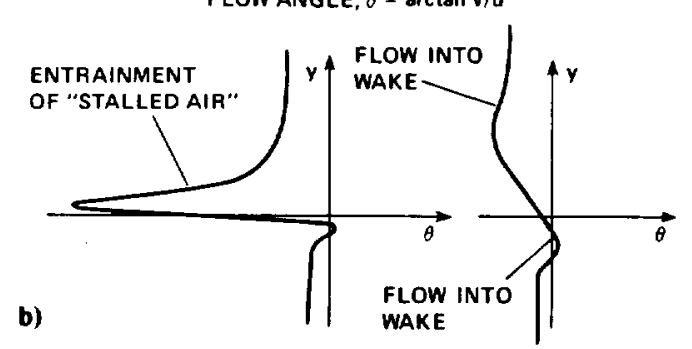

Fig. 9 Trailing-edge models for stall conditions: a) model of Ref. 14 for GA(W)-1 airfoil section; b) proposed model for NACA 64A010 girfoll section.

also at $\alpha=3.5 \mathrm{deg}$ that the trailing edge $C_{p}$ first starts to deviate from the value at $\alpha=0$ deg, decreasing with further increase in angle. Mean velocity data ${ }^{\prime}$ obtained near the trailing edge for $\alpha=3.5 \mathrm{deg}$ indicated that the flow was attached at the trailing edge (i.e., the minimum streamwise velocity at $x / c=1.02$ was not significantly lower than that for $\alpha=0$ deg $)$. The nominally low value for $\left(-\overline{u^{\prime} v^{\prime}} / u_{e}^{2}\right)_{\text {max }}$ at $\alpha=3.5$ deg suggests the presence of only a small separation bubble, whereas the large and constant level of the maximum turbulent shear stress for $\alpha=5 \mathrm{deg}$ and greater must be the result of separation extending into the wake with the attendant development of a free-shear-layer character to the flow. These shear layers, however, differ significantly in thickness. As seen in Fig. 2, the lift continued to increase for $\alpha>5$ deg even though the flow is separated from the base of the shock wave to the trailing edge. 


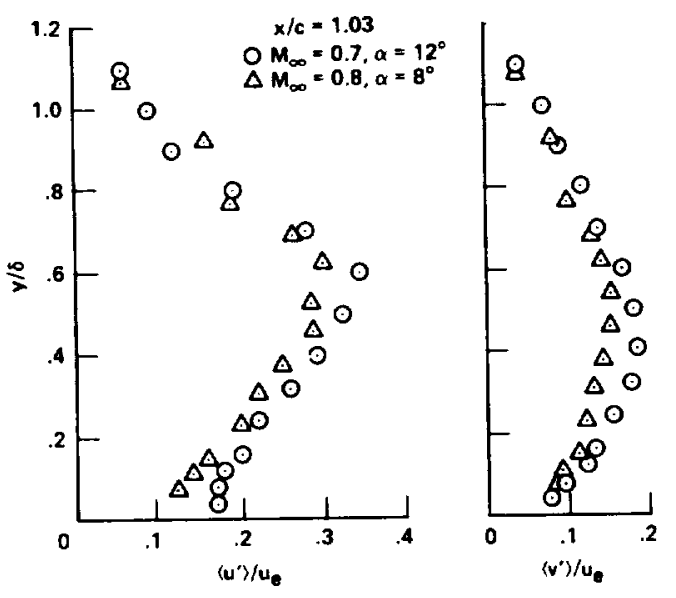

Fig. 10 Shear-layer turbulence intensities at trailing edge.

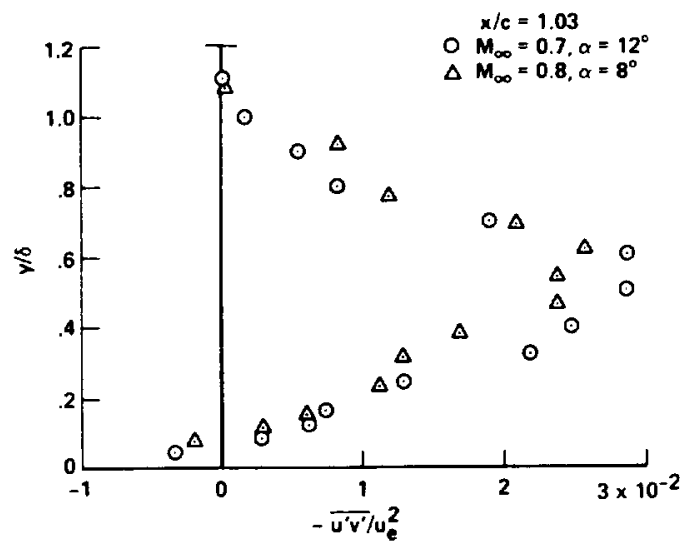

Fig. 11 Shear-layer turbulent shear stresses at trailing edge.

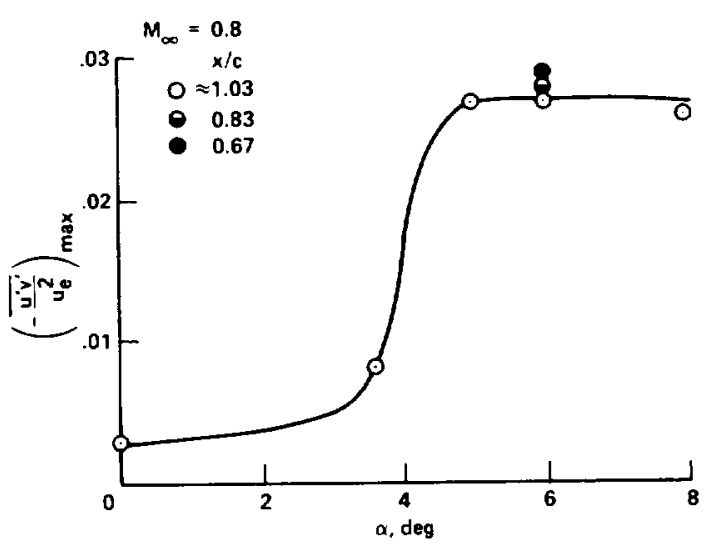

Fig. 12 Turbulent shear stress development at trailing edge.

From Figs. 6 and 11 it is seen that the point of maximum shear stress occurs near the location where the strain rate $\partial \bar{u} / \partial y$ is the greatest. This would imply that an eddy viscosity description may be valid in describing the shear behavior. In Fig. 13, Prandtl's mixing length $l$, scaled to the boundarylayer thickness

$$
\frac{l}{\delta}=\frac{\left(-\overline{u^{\prime} v^{\prime}} / u_{e}^{2}\right)^{1 / 2}}{\partial\left(\bar{u} / u_{e}\right) / \partial(y / \delta)}
$$

in the middle portion of the layers, is compared to the model suggested by Escudier ${ }^{17}$ for turbulent boundary layers. Because accurate mean velocity gradients are extremely

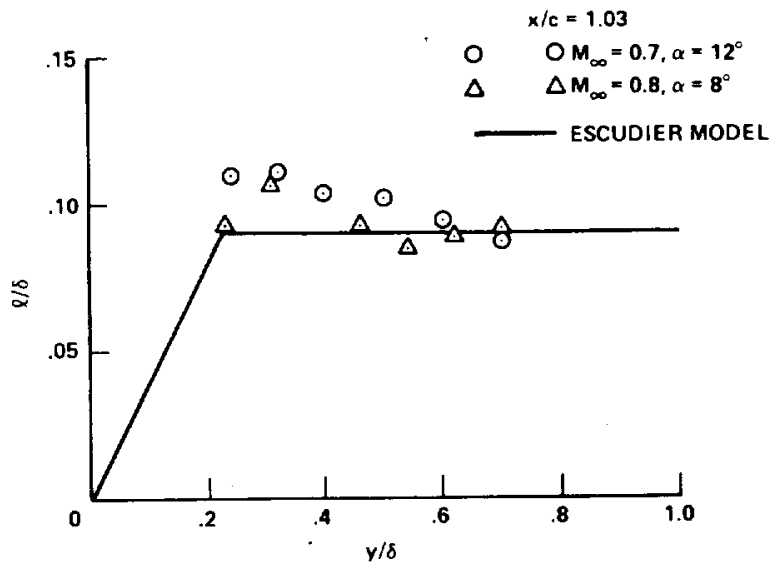

Fig. 13 Mixing length distributions across trailing-edge shear layers.

difficult to obtain from experimental data, especially when the data are sparse, $l$ was determined only in the region where the slope was nearly constant and, thus, best defined. The value of $1 / \delta \approx 0.1$ for the central portion of the shear layer (Fig. 13) was also found to hold near the trailing edge at all three angles of attack studied in Ref. 1 . Spencer and Jones also observed $l / \delta$ to be nearly constant with a value near 0.1 across the mixing layer in their low-speed experiment. Apparently, the nondimensional strain rate $\left(\partial \bar{u} / u_{e}\right) /(\partial y / \delta)$ was lower in that experiment which would account for the lower shear stress for the same $/ / \delta$. In Ref. $1, l / \delta$ increased rapidly beyond the trailing edge, approaching the value of 0.18 that has been observed for far wakes. ${ }^{18}$

Although the shear layer for the $\alpha=8$ and 12 deg cases have had entirely different flow histories, equilibrium between the mean flow and the turbulent fluctuations appears to prevail in the central portion of these layers at the trailing edge. This condition was found to still hold at the furthermost upstream measurement station $(x / c=0.67)$ for $\alpha=6 \mathrm{deg}$. At this stage of the flow development, the flow has apparently lost all memory of its origin, as it does in a flow with self-similarity. The data indicate that, at least in certain portions of the flow, a simple eddy viscosity turbulence model should be adequate, even for the extreme separation cases of this study.

\section{Summary}

Unique flowfield measurements were made for a $64 \mathrm{~A} 010$ airfoil section at transonic conditions with either shockinduced or leading-edge separation present. Through the application of holographic interferometry to define global flow features and laser velocimetry to describe the details of the viscous flow regions, the complicated viscous-inviscid interactions that occur on a stalled airfoil at transonic speeds could be addressed.

A comparison of static pressures at the outer edge of the shear layers, as obtained from infinite-fringe interferograms with measured surface pressures, revealed the static pressure changes across those shear layers to be very small. Thus, the Kutta condition appears to be valid for these extremely separated flow conditions.

The viscous layer was found to be about twice as thick at the trailing edge for the leading-edge separation than it was for the shock-induced separation case. However, by scaling the vertical distance by the boundary-layer thickness, and the mean velocity and turbulent fluctuations to the edge velocity, the two sets of data differed very slightly. Cole's wake function profile described the mean profiles reasonably well. In the near wake, flow-angle data reveal substantial entrainment of the slow-moving fluid above the airfoil's surface into the high-energy lower surface boundary layer.

The properties of the two shear layers are similar to those observed in low-speed, two-stream mixing experiments, the 
maximum turbulence levels being about $50 \%$ higher and the shear stress, scaled to $u_{e}^{2}$, being about twice that of the lowspeed shear layer with a zero pressure gradient. For the present data, $/ / \delta$ equals approximately 0.1 in the central portion of the shear layers, as has also been observed for lowspeed shear layers. The maximum strain rate, $\left[\left(\partial \bar{u} / u_{e}\right) / \partial y / \delta\right]_{\max }$, appears to be about $50 \%$ larger for the present data, which accounts for a value for $\left(-\overline{u^{\prime} v^{\prime}} / u_{e}^{2}\right)_{\max }$ that is greater by a factor of 2 than that for low-speed conditions. An interesting feature of the data presented here is the near constant value for $\left(-\overline{u^{\prime} v^{\prime}} / u_{e}^{2}\right)_{\max }$ at the trailing edge, once separation has progressed to that point. The results indicate that even for these massive separations, an eddy viscosity turbulence model should be adequate over a substantial part of the flow.

Further experiments should be performed to study the development of the flow in order to determine when local equilibrium between the mean flow and the turbulent fluctuations first establishes itself. Also, the near-wake development should be explored in more detail; this can be accomplished by simply mounting the airfoil section farther forward in the test section. The exploration of the mean and turbulent flow behavior at the point of separation will probably have to be done using basic flow models with thick initial boundary layers; this is because the airfoil boundary layers are extremely thin just prior to separation when separation occurs far forward on the airfoil.

\section{References}

${ }^{1}$ Johnson, D.A. and Bachalo, W.D., "Transonic Flow About a Two-Dimensional Airfoil - Inviscid and Turbulent Flow Properties," AIAA Journal, Vol. 18, Jan. 1980, pp. 16-24.

${ }^{2}$ Seetharam, H.C. and Wentz, W.H., Jr., "Experimental Investigation of Subsonic Turbulent Separated Boundary Layers on an Airfoil," Journal of Aircraft, Vol. 14, Jan. 1977, pp. $51-55$.

${ }^{3}$ Coles, D. and Wadcock, A.J., "Flying Hot-Wire Study of Flow Past an NACA 4412 Airfoil at Maximum Lift," AIAA Journal, Vol. 17, April 1979, pp. 321-329.

${ }^{4}$ Young, W.H., Jr. and Hood, D.R., "Comparison of Two Flow Surveys Above Stalled Wings," AIAA Paper 79-0332, AIAA 17th Aerospace Sciences Meeting, New Orleans, La., 1979.
${ }^{5}$ Seegmiller, H.L., Marvin, J.G., and Levy, L.L., Jr., "Steady and Unsteady Transonic Flows," AIAA Journal, Vol. 16, Dec. 1978, pp. 1262-1270.

${ }^{6}$ Alber, I.E., Bacon, J.W., Masson, B.S., and Collins, D.J., "An Experimental Investigation of Turbulent Transonic Viscous-Inviscid Interactions," AIAA Journal, Vol. 11, May 1973, pp. 620-627.

${ }^{7}$ Lo., C.F., Heltsley, F.L., and Altstatt, M.C., "A Study of Laser Velocimeter Measurements in a Transonic Flow," AlAA Paper 76333, AIAA 9th Fluid and Plasma Dynamics Conference, San Diego, Calif., 1976.

${ }^{8}$ East, L.F., "The Application of a Laser Velocimeter to the Investigation of Shock-Wave Boundary-Layer Interactions," $A p$ plications of Non-Intrusive Instrumentation in Fluid Flow Research, Saint-Louis, France, AGARD Conference Proceedings 193, May 1976.

${ }^{9}$ Bachalo, W.D., Modarress, D., and Johnson, D.A., "Experiments on Transonic and Supersonic Turbulent Boundary Layer Separation," AIAA Paper 77-47, AIAA 15th Aerospace Sciences Meeting, Los Angeles, Calif., 1977.

${ }^{10}$ Bachalo, W.D, and Johnson, D.A., "An Investigation of Transonic Turbulent Boundary Layer Separation Generated on an Axisymmetric Flow Model," AIAA Paper 79-1479, AIAA 12th Fluid and Plasma Dynamics Conference, Williamsburg, Va., 1979.

"McLaughlin, D.K. and Teiderman, W.G., "Biasing Corrections for Individual Realization of Laser Anemometer Measurements in Turbulent Flows," Physics of Fluids, Vol. 16, Dec. 1973, pp. 20822088.

${ }^{12}$ Trolinger, J.D., "Laser Instrumentation for Flow Field Diagnostics," AGARDograph 186, March 1974.

${ }^{11}$ Coles, D., "The Law of the Wake in the Turbulent Boundary Layer," Journal of Fluid Mechanics, Vol. 1, Part 2, July 1956, pp. 191-225.

${ }^{14}$ Zumwalt, G.W. and Naik, S.N., "An Analytical Model for Highly Separated Flow on Airfoils at Low Speeds," Wichita State University, Wichita, Kan., Aeronautical Report 77-2, May 1977.

is Leipmann, H.W. and Laufer, J., "Investigations of Free Turbulent Mixing," NACA TN-1257, 1947.

${ }^{16}$ Spencer, B.W. and Jones, B.G., "Statistical Investigation of Pressure and Velocity Fields in Turbulent Two-Stream Mixing Layer," AIAA Paper 71-613, AIAA 4th Fluid and Plasma Dynamics Conference, Palo Alto, Calif,, 1971.

${ }^{17}$ Escudier, M.P., "The Distribution of the Mixing Length in Turbulent Flows Near Walls," Imperial College, London, Rept. TWF/TN/1, 1965.

${ }^{18}$ Schlichting, H., Boundary Layer Theory, McGraw-Hill Book Co., New York, 1955, Chap. 23, p. 602. 